\title{
Variations
}

Variations

Revue internationale de théorie critique

$17 \mid 2012$

Critique du travail

\section{Critique du travail et révolution du capital}

Jacques Wajnsztejn

\section{(2) OpenEdition}

Journals

Édition électronique

URL : http://journals.openedition.org/variations/362

DOI : 10.4000/variations.362

ISSN : 1968-3960

\section{Éditeur}

Les amis de Variations

\section{Référence électronique}

Jacques Wajnsztejn, « Critique du travail et révolution du capital », Variations [En ligne], 17 | 2012, mis en ligne le 15 octobre 2012, consulté le 30 avril 2019. URL : http://journals.openedition.org/ variations/362 ; DOI : 10.4000/variations.362

Ce document a été généré automatiquement le 30 avril 2019.

Les ami•e•s de Variations 


\title{
Critique du travail et révolution du capital
}

\author{
Jacques Wajnsztejn
}

1 Quand un cycle révolutionnaire atteint ses limites, c'est sur ces limites que prospère le cycle suivant, qu'il s'agisse d'un cycle contre-révolutionnaire comme celui des années 1920-1930 succédant aux révolutions russes et allemandes ou qu'il s'agisse d'un cycle de restructuration comme celui qui succède aux bouleversements historiques des années 1960-1970. Dans les deux cas il procède par retournement de ces limites à son profit.

2 La critique et le mouvement de refus du travail ont ainsi été retournés au profit du capital. L'absentéisme et le turnover ouvriers sont devenus flexibilité et précarité patronales. Les ouvriers à la chaîne (OS) ont été en partie remplacés par des robots et les grandes forteresses ouvrières ont été démantelées. La restructuration s'est faite à partir d'une organisation en réseau qui mêle quelques centres productifs "dégraissés " ( reengineering) et une toile d'araignée de filiales et d'entreprises sous-traitantes. On est passé d'un tissu industriel usinier à l'entreprise-réseau.

3 La société du travail est arrivée à son terme... Et il n'y a pas à le regretter, mais le rapport social capitaliste n'a pas dit son dernier mot. Il essaie de compenser toute la masse de travail vivant devenu inutile en transformant toute activité, y compris celles qui restaient à sa marge, en emplois utiles (développement du travail «domestique » rétribué, de la garde d'enfants et de personnes âgées, du secteur social et culturel, etc.). Il ne faut donc pas confondre inessentialisation de la force de travail et fin du travail.

4 La société capitalisée combine tendance à la valorisation sans le travail et maintien du travail comme élément de domination plus que d'exploitation.

\section{Qu'est-ce que le travail ?}

Le travail est une activité séparée des autres activités humaines. Elle n'est plus l'activité vitale des hommes qui exprime un certain rapport au monde en s'y incluant, mais un rapport à une nature devenue extérieure. Cette activité travail présente le double 
caractère d'être tendance à la domination sur cette nature et en même temps d'être l'expression d'une domination des hommes sur d'autres hommes. Ce phénomène est bien antérieur à l'exploitation capitaliste. La domination, c'est ce qui est commun à toutes les sociétés étatisées - y compris donc à celle que nous appelons la « société capitalisée ».

Mais cette domination prend la forme particulière de l'exploitation de la force de travail libre comme base d'accroissement du profit dès la phase de domination formelle $d u$ capital (de la première révolution industrielle à 1914) puis dans la première phase de la domination réelle du capital ${ }^{1}$ (années 1920/30). Ce n'est que dans ce cadre que le travail est placé au cœur des rapports sociaux et que l'on a affaire à un "mode de production ", alors que dans les systèmes pré-capitalistes, même si la majorité de la population travaille, que le capital s'accumule de façon primitive et que la valeur circule, le travail se trouve encore inclus dans un ensemble plus large de rapports définis par des liens de dépendance personnels. Comme le disait Marx: les rapports entre les hommes sont seulement médiés par les choses, alors que le capitalisme apparait comme un rapport entre les choses médié par des hommes. Il s'ensuit que la disparition de la figure du bourgeois ou même du capitaliste moderne ne conduit pas automatiquement à la disparition du travail comme l'ont montré a contrario les exemples de la révolution russe et de la guerre civile espagnole d'un côté et l'avènement de la « société capitalisée » de l'autre.

7 Il ne s'agit donc pas de « libérer » le travail de son carcan capitaliste, ce qui reviendrait à ne critiquer le travail que sous sa forme de travail salarié et donc à faire du concept de travail (le «travail en général »), l'expression unique du métabolisme de l'homme avec la nature ${ }^{2}$.

L'activité ne se cache pas non plus sous le travail dans la mesure où l'activité est aujourd'hui présente partout en tant qu'activité en crise et activité de crise. Dans cette crise, travail et activité s'entrecroisent puisque toute activité semble être transformée en travail car rien ne doit échapper à la généralisation de la société capitalisée, mais le travail reste quand même une opportunité pour l'activité (perruque, savoir-faire, innovations, inventions, reconnaissance individuelle, relations sociales) malgré le caractère d'aliénation propre au rapport social capitaliste.

Si la crise de reproduction des rapports sociaux est devenue à ce point profonde, c'est que c'est le capital lui-même qui est aujourd'hui à la pointe de la remise en cause de la centralité du travail. Sa pratique ne passe pas par une abolition consciente et totale du travail, mais par des transformations concrètes du procès de production qui produisent une tendance à l'inessentialisation de la force de travail, une tendance à la valeur sans le travail par substitution du travail mort (capital fixe) au travail vivant (capital variable). Le travail n'étant plus au centre du procès de valorisation, il ne peut plus être central au sein de la société capitalisée et ses revendications s'en trouvent délégitimées. Mais il la structure encore à travers les catégories du salariat et ses valeurs, telle la "valeurtravail » comme disent les dirigeants politiques qui confondent le travail comme valeur idéologique avec le travail comme producteur de valeur matérielle. Comme dans toutes les périodes transitoires, grosses de bouleversements, l'ancien côtoie le nouveau.

10 Pour nous, le travail est produit historiquement par la prédominance de la production matérielle qu'impose, pour reprendre une formule d'Adorno, un rapport de domination à la nature extérieure et non plus un simple échange de substance avec la nature. Cette activité principale centrée sur une prédominance de la production matérielle induit la 
séparation d'avec les autres activités et crée la possibilité de l'accumulation d'un surproduit au-delà des besoins collectifs de la communauté d'origine. C'est sur cette base que progressivement l'activité travail va devenir économie de la société et que les théories sur la valeur-travail et le travail productif s'élaboreront au sein de ce que Marx a appelé la « domination formelle du capital ».

Dans cette phase, on peut dire que le travail vivant est véritablement au centre des rapports sociaux, même si évidemment, il ne trouve son emploi que dans l'existence et la médiation de l'autre pôle du rapport social, à savoir la part du capital constitué par le travail mort. Mais avec le passage à la «domination réelle du capital» (dès 1933 aux États-Unis et à partir de 1945 en Europe, puis pleinement à partir de la fin des années 1960-70), le procès de valorisation tend à s'autonomiser du procès de travail. C'est sur cette base que la critique du travail va prendre une telle importance avec par exemple les thèses situationnistes et celles de la revue Socialisme ou Barbarie au niveau théorique, le Mai 1968 français et le mouvement italien de refus du travail dans les grandes usines du nord de 1969 à 1973 au niveau pratique. Mais la théorie critique n'étant jamais suspendue en l'air, ce thème de la critique du travail en général - et non pas simplement du travail salarié - présuppose que le travail concret, le travail vivant, n'est plus la source essentielle de valorisation.

Toutefois, cet angle d'attaque qu'a constitué la critique du travail ne doit pas faire oublier que ce qui est fondamental, en dernier ressort, c'est la critique du capital en tant que totalité, d'autant que la restructuration qui s'opère dans les entreprises à ce moment-là, comme le phénomène parallèle de globalisation/mondialisation, marquent une avancée dans un processus de totalisation que nous percevions mal à l'époque.

\section{Travail et pratiques anti-travail}

13 Aujourd'hui, la pratique anti-travail est l'expression d'une subjectivité particulière qui, en tant que telle, n'a pas plus d'effet pratique sur les rapports sociaux que le fait de continuer à bien faire son travail, au moins dans les périodes qui n'offrent pas de possibilité de passer à autre chose. Cette contradiction est présente dès le début du mouvement révolutionnaire comme on peut le voir dans l'opposition entre anarchistes individualistes et anarcho-syndicalistes. Mais la position anti-travail d'individus comme Darien ou Libertad reste minoritaire car elle s'affirme à contre-courant historique du processus qui transforme les prolétaires des «classes dangereuses » en ouvriers. Cette critique est donc énoncée de l'extérieur du rapport social capitaliste tel qu'il est en train de s'affirmer à l'époque sur la base du rapport de dépendance réciproque entre capital et travail. C'est cette même extériorité qui caractérise la critique anti-travail que mène l' Internationale Situationniste dans les années 1960. Critique externe parce qu'elle ne surgit directement ni de la théorie communiste, ni des pratiques de lutte de classes de l'époque. Elle se fonde sur une analyse plus large des contradictions du capital et de rapports sociaux en train d'être révolutionnés par l'utilisation des techno-sciences, de la communication, de la consommation, etc.

14 Si cette critique devient possible et audible après plus d'un demi-siècle d'idéologie marxiste forcenée du travail, ce n'est pas principalement du fait qu'elle trouve un écho dans une petite frange de la jeunesse ouvrière ou étudiante de l'époque, mais parce que le rapport social capitaliste a commencé sa propre critique du travail. La critique subjective trouve ici ses conditions objectives. On voit donc resurgir parallèlement P. Lafargue avec 
son Droit à la paresse et l'utopie d'une automation totale rendue possible par un haut degré d'accumulation, prémisse supposée d'une fin du travail en tant qu'activité aliénée. Toutefois la position de l'IS n'est pas différente de celle des autres courants marxistes de l'époque: le progrès technique est glorifié parce que nous sommes toujours dans la croyance en la libération ou la désaliénation par le Progrès ${ }^{3}$.

Dans les groupes informels auxquels je participe à l'époque, la critique anti-travail s'appuie sur les récentes pratiques prolétariennes d'absentéisme, de sabotage, de grèves dures sans revendications particulières, qui sont courantes dans la première moitié des années 1970. Cette critique est reliée, au niveau théorique, à l'idée d'auto-négation du prolétariat. C'est la tentative la plus avancée, à l'époque, pour essayer de résoudre, au moins au niveau théorique, la fameuse contradiction dans laquelle Debord et l'IS sont tombés, à savoir d'un côté porter la critique du travail à son plus haut niveau (" $\mathrm{Ne}$ travaillez jamais ») et de l'autre faire l'apologie des conseils ouvriers.

Comprendre la production du capital comme rapport social contradictoire implique de refuser une démarche du type ou/ou (travail ou paresse par exemple) car le travail étant une composante du rapport social capitaliste, affirmation et négation coexistent dans le même individu prolétaire.

Le refus du travail ne peut donc être une ligne de démarcation car ce n'est ni une position (c'est l'expression d'une subjectivité), ni une revendication (qui présuppose le travail), ni une action précise (comme l'abolition du travail dans la révolution). C'est un mouvement de révolte historiquement situé, une forme concrète de lutte de classes, mais conjoncturelle. C'est la radicalisation de ce mouvement qui seule aurait pu créer les conditions révolutionnaires et l'auto-négation du prolétariat vers la communauté humaine (une "révolution à titre humain $\left.{ }^{4} »\right)$. En son absence, le mouvement va refluer rapidement parce que ce n'est pas une position collective tenable.

Le refus du travail n'est pas un élément de la conscience de classe, car celle-ci comprend l'affirmation du travail comme puissance potentielle de la classe productive et capacité à transformer le monde. Le refus du travail est rupture avec ce processus d'affirmation et ne se développe que dans les moments de crise de la reproduction des rapports sociaux. C'est pour cela que la controverse de l'époque entre J. Zerzan (le refus du travail comme désir radical) et Ch. Reeve (le refus du travail comme expression d'un rapport de force favorable) reste vaine si on ne saisit l'opposition qu'au niveau théorique, alors qu'elle exprimait un point de basculement de l'Histoire : vers l'abolition du capital et du travail par les prolétaires ou vers une défaite rendant impossible tout nouveau rapport de force favorable parce qu'elle va conduire à la fin de la centralité du travail pour le capital ${ }^{5}$.

Nous savons aujourd'hui qui a gagné, mais comme à toute chose malheur est bon, il y a au moins un avantage à la situation actuelle, c'est que le travail ne peut plus s'y affirmer, ni en tant que porté par une classe ni en tant qu'essence ou mesure de la valeur. L'ancienne contradiction, interne au prolétariat, entre affirmation et négation devient caduque. L'affirmation du travail qui constituait en partie la classe ouvrière s'est dissoute dans la désindustrialisation, les luttes pour la retraite, la résignation au travail-revenu constitutive de l'individu-salarié indifférencié. Il n'y a plus de place pour une vision messianique du prolétariat comme classe à qui il aurait été attribué la double tâche d'une transformation pratique du monde en collaboration avec la bourgeoisie industrieuse et d'une révolution prolétarienne antagonique au capital. 
20 Il n'y a donc plus d'identification absolue à un travail qui aurait une positivité en soi, mais prise de distance et c'est ce qui est potentiellement subversif. Cette mise à distance qui dépasse la question du travail (salarié ou non) et qui fait que l'individu ne se confond jamais avec l'objet de son activité est justement ce qui définit l'humain et fait sa spécificité, mais elle n'apparaît pas en continu. Cette propriété de l'humain est aussi ce qui permet de comprendre le mystère de la participation au travail. Le travail n'est pas que domination et exploitation; s'il est bien défini par le fait d'être une activité aux ordres, il est aussi expérimentation, savoir-faire et parfois même passion de l'activité. Bien sûr, plus on s'éloigne du travail de type artisanal et pour tout dire des formes concrètes du travail, plus cet aspect devient secondaire ou disparait.

21 Du point de vue de la clarté du langage il vaudrait mieux faire la distinction entre la valeur-travail (ou exactement la théorie de la valeur-travail) et l'idéologie du travail comme valeur, sinon on occulte l'aspect économique et objectif de la chose et on ne peut comprendre que l'une ne va pas sans l'autre, qu'il ne s'agit pas d'un masque. Obnubilés par la représentation (le fétichisme) et le résultat sur le marché (la marchandise), certains, centrés sur la forme-valeur et le travail abstrait qui en serait le contenu, oublient que le point de départ, c'est l'activité de transformation de la nature extérieure à travers la production matérielle (mais plus seulement aujourd'hui) au sein de rapports sociaux déterminés qui ne font l'objet que d'une contestation idéologique. Sinon on ne comprendrait pas pourquoi le travailleur libre du capitalisme continuerait à travailler si le travail n'est que tripalium. Le salaire ne représente en effet qu'une dimension de l'affaire et quand les travailleurs d'ArcelorMittal ou les ouvrières de Lejaby avancent que pourtant ils sont encore productifs et utiles, ce n'est pas essentiellement parce qu'ils espèrent convaincre leurs patrons ou l'État, mais parce qu'ils croient encore en la théorie de la valeur-travail (ce sont eux qui produiraient toujours et dans les mêmes proportions, la richesse sociale) et qu'ils défendent le travail comme valeur par rapport à la spéculation et à l'abominable finance. Néanmoins, ils ne peuvent plus affirmer le travail et c'est pour cela qu'ils hésitent moins à employer des méthodes de lutte en rapport avec leur désespoir. Un désespoir de travailleurs qui se définissent encore principalement par leur travail alors qu'ils sont amenés à ne plus travailler ou pour certains, à occuper un emploi qui ne leur apparaît plus comme un travail, mais comme une simple activité de survie ou un job.

Poser un au-delà du travail qui s'appuie seulement sur la force de la négation c'est repartir sur les mêmes bases que dans la période précédente. C'est encore buter sur la question de l'activité, des rapports sociaux, toutes choses qui ne se posent pas uniquement en termes de négation sous prétexte que le Marx hégélien a parlé du « travail du négatif ». Et le développement actuel de pratiques alternatives ne se réduit pas à du pur réformisme.

On ne peut sauver la place centrale des producteurs dans le programme prolétarien en appelant à une unité entre producteurs et consommateurs. Cela correspond peut-être à un programme révolutionnaire qui a inscrit à son fronton théorique : " abolition de toutes les séparations ", mais qu'est-ce que cela peut bien vouloir dire si on est d'accord pour reconnaître justement que le capital a, en tendance, supprimé les producteurs et unifier son processus ? Là encore on ne peut pas plaquer les recettes du programme prolétarien sur une situation qui n'est plus celle de la lutte des classes entre travail et capital. C'est la thèse de l'auto-négation du prolétariat qui est caduque. Cette thèse, fondamentalement aclassiste et donc impossible à tenir à partir d'un discours classiste, le devient encore plus 
à partir du moment où le capital a englobé l'antagonisme entre les classes, à partir du moment où c'est lui qui cherche à nier la dépendance réciproque entre les deux pôles du rapport social, bref, à s'auto-présupposer comme le montre aujourd'hui le rôle du capital fictif ${ }^{7}$ comme auto-présupposition du profit (A-A' tend à se réaliser sans passer par $\mathrm{M}, \mathrm{A}$ M-A').

Il y a problème dès qu'on projette un programme sans tenir compte des transformations et donc des conditions dont il faut partir. On retrouve ce problème quand certains posent la question de l'appropriation de la richesse produite. La société capitalisée ne se caractérise plus par ce que Marx appelait une gigantesque accumulation de marchandises. Aujourd'hui ce qui s'accumule, ce sont de plus en plus des choses non appropriables qui posent la question de leur usage. C'est parce que nous ne sommes plus dans le cadre de la prédominance de la production matérielle et le schéma de la dialectique des classes, que «l'expropriation des expropriateurs » et le « Rien n'est à eux tout est à nous" ne sont plus que des slogans nostalgiques et non pas des outils d'intervention politique. Ainsi, la réactivation du mot d'ordre de «prise sur le tas », n'a littéralement aucun sens en dehors de celui d'une ponctuelle action de pillage dans une révolte spontanée.

D'ailleurs, beaucoup de choses qui n'étaient utiles que par rapport à la logique du capital disparaîtront, contrairement à ce que semble penser le courant « communisateur ».

\section{La crise du travail} luttes et pratiques de refus du travail, dans un cycle de luttes révolutionnaires concernant l'ensemble des pays occidentaux. Mais depuis le retournement de ce cycle dans la crise qui s'amorce au milieu des années 1970, ce sont les restructurations industrielles et leurs conséquences sur les rapports sociaux qui produisent une véritable critique pratique du travail. Elle s'accompagne d'un mouvement contradictoire: en tendance le travail n'est plus au centre de la société capitalisée, mais sous la forme de l'emploi, il reste ce qui définit les statuts et les droits des individus. Cette contradiction est bien rendue par plusieurs phénomènes tels que : l'ambiguïté du slogan d'agir contre le chômage $(A C)$ : «Le travail est un droit, le revenu est un dû». La déclaration de Jospin contre l'assistanat pendant la lutte des chômeurs de 1998 en France, la vogue de l'idéologie blairiste au sein de la social-démocratie européenne, les déclarations de Sarkozy et Royal pendant les présidentielles de 2007 visant à remettre le travail comme 
valeur au centre de la société expriment des tendances fortes à maintenir le travail comme idéologie et valeur en l'absence de toute résolution pratique de la contradiction.

Simulation de situations de travail, traitement social du chômage, stages, retour au travail forcé par la suppression ou la dégressivité de diverses allocations sont les moyens employés pour réaliser ce tour de force du maintien d'une société du travail sans travailleurs comme $\mathrm{H}$. Arendt le signalait déjà.

Dans ce contexte qui est à la fois déréalisation du travail vivant concret dans des emplois où triomphent activités virtuelles et idéologie du travail afin de justifier une reproduction en l'état des rapports sociaux, il devient difficile de continuer à parler en termes de rapports de force pour signifier que perdure la lutte des classes. En effet pour qu'il y ait rapport de forces faut-il encore qu'il y ait des forces en présence et que celles-ci révèlent un antagonisme historiquement irréductible entre les classes dans la perspective révolutionnaire où une opposition entre intérêts différents comme dans la vision socialdémocrate.

31 Par exemple, le mode de régulation fordiste consistait en un échange de hausses de productivité contre des hausses du pouvoir d'achat dans le cadre du partage de la valeur ajoutée, échange ponctué de négociations et même de conflits contrôlés par les syndicats pour des ajustements nécessaires au moment des renouvellements de conventions collectives ou pour l'échelle mobile des salaires. Ce compromis d'ordre global n'a pas empêché que se développent de grandes luttes d'OS dans tous les pays industrialisés occidentaux. Or les précaires d'aujourd'hui ne sont pas les OS d'hier et les luttes d'hier ne sont pas celles d'aujourd'hui. Le contexte général est plus favorable aux entreprises et c'est surtout le caractère antagonique qui a du mal à s'exprimer à partir du moment où les anciens intérêts de classes semblent s'évanouir devant des intérêts communs face à la concurrence ou à l'étranger.

«L'épreuve de force " n'est plus envisagée que de manière localiste, désespérée, par exemple au niveau d'une usine menacée de fermeture. C'est une résistance qui peut être forte comme chez Continental et d'autant plus forte qu'elle ne s'illusionne pas sur les possibilités de sauver l'emploi. En effet, la lutte s'est concentrée sur le fait de monnayer la valeur d'une force de travail devenue inemployable. Mais c'est une résistance isolée.

Cette crise du travail est reportée en premier lieu sur une partie de la force de travail, celle qui est rendue surnuméraire de façon absolue par l'introduction d'un progrès technique destructeur des anciennes qualifications définies dans la communauté du travail par un lien professionnel entre travail vivant et capital technique. Ce qui les remplace, ce sont de nouvelles compétences déterminées par le seul patronat ou l'Étatpatron et attribuées à titre individuel. Quant à l'autre partie de la force de travail, jugée essentielle - mais pour combien de temps? - parce que mieux formée et plus polyvalente, elle est peu ou prou assimilée à du capital en tant que "capital humain " comme disent les américains ou en tant que "ressource humaine" comme on dit en France.

En second lieu, elle est reportée aussi sur le capital global dont le représentant au niveau national est l'État, chargé de redistribuer les richesses. C'est la façon qu'a l'État, de répondre à la revendication de "partage de richesses » et d'arbitrer entre différentes solutions qui toutes doivent tenir compte du fait que le revenu se détache de plus en plus du travail réel effectué. Dans cette mesure, le «salaire minimum» n'est plus depuis longtemps, le prix de la reproduction de la force de travail. Seillères, l'ex-patron du 
MEDEF, le déclare sans fioriture: «Les employeurs paient les salaires que le marché impose; si la société estime que ce n'est pas assez pour vivre, qu'elle complète!». La « prime pour l'emploi » va dans ce sens car elle s'oppose à la fois au crédit d'impôt de la droite libertarienne appliqué aux État-Unis et à la revendication d'un revenu minimum universel revendiqué par les gauches alternatives. Elle n'est donc pas une tentative de mobilisation productive (de survaleur) des chômeurs tendant à dissoudre le précariat dans le workfare. L'échec du RSA va dans le même sens : s'il joue son rôle d'assistance avec l'augmentation du nombre d'allocataires, il ne joue pas son rôle d'incitation au travail parce que $60 \%$ des personnes qui pourraient le faire jouer en complément d'un travail précaire n'effectuent pas les formalités pour en bénéficier.

\section{Crise du travail et précariat}

35 La notion fourre-tout de "précaire " prend une dimension de plus en plus idéologique, comme on peut le voir avec l'idée d'une centralité du précariat ${ }^{8}$. Cela se manifeste de deux façons :

36 - premièrement, les chiffres statistiques des flux de main-d'œuvre (entrants et sortants) sont privilégiés par rapport à ceux des stocks (fixes-garantis), pourtant ces derniers sont nettement plus nombreux en valeur absolue. Sous prétexte que les flux seraient aujourd'hui majoritairement précarisés, il en découlerait que ce mouvement est irréductible et donc qu'à terme, c'est toute la force de travail qui se retrouverait dans cette situation, indiquant par là une nouvelle régulation des rapports de travail et une nouvelle unification des statuts par le bas. Cela conduit aussi à assimiler les jeunes, les femmes et les immigrés à une figure majeure de la population active dont la caractéristique serait d'être précaire et de subir cette précarité.

37 C'est ignorer que les jeunes diplômés même s'ils mettent aujourd'hui plus de temps qu'hier à trouver un travail stable et correspondant à leur niveau d'études, ne peuvent être confondus avec les $20 \%$ de jeunes qui sortent sans aucun diplôme ou qualification du système scolaire; c'est oublier aussi que le niveau de diplôme et de qualification des femmes augmente de façon continue; c'est oublier enfin que pour les immigrés de seconde ou troisième génération la mobilité sociale ascendante n'est pas un vain mot. Il ne faut pas confondre généralisation du précariat et maintien de formes de discrimination à l'embauche.

- deuxièmement, cela conduit à associer absolument flexibilité et précarité comme s'il s'agissait d'une évolution univoque des statuts alors que les pratiques d'absentéisme et de turnover dans les années 1960-1970 comme le recours des jeunes au travail intérimaire et plus généralement à l'intermittence du travail aujourd'hui, prouvent que ces formes représentent aussi une résistance au travail salarié. Certains aspects du mouvement des intermittents du spectacle vont dans ce sens, même s'il ne faut pas les mythifier et en faire le modèle de conquête d'une nouvelle autonomie, celle du salarié du cognitariat comme nouvelle figure du travail des années 2000 pour parler comme les néo-opéraïstes.

Du côté patronal les choses ne sont pas fixées non plus. La majorité actuelle du patronat français souhaite une "refondation" sociale remettant en cause les types actuels de contrats de travail en vue d'une meilleure flexibilité, mais ce projet provient de personnalités qui ne sont pas issus du monde de l'industrie, mais plutôt de la finance ou des assurances. À l'inverse, les syndicats patronaux de la métallurgie et de l'industrie 
lourde cherchent plutôt à investir dans la ressource humaine, alors que de leur côté, les PME/PMI qui sont pourtant les plus grosses consommatrices de main-d'œuvre précaire, souhaiteraient attirer durablement des salariés, sans avoir les moyens (conditions de travail, statuts et salaires) de les fidéliser.

- enfin, troisièmement, à masquer la division objective qui sépare les salariés, mêmes précaires, des chômeurs et particulièrement des chômeurs de longue durée et des laissés pour compte du salariat. Cette tendance trouve son actualisation dans des slogans tels celui des Occupy de New-York: " Nous sommes les $99 \%$ », comme si une classe d'exploités et de dominés s'était recomposée suite à la déstructuration et à l'atomisation de l'ancienne classe ouvrière.

41 Ces simplifications et ces méprises s'accompagnent d'une vision traditionnelle et datée de l'État comme simple superstructure dépendant d'une infrastructure économique qui lui échapperait de plus en plus dans le cadre de la globalisation/mondialisation. C'est oublier qu'il n'y a pas que le capital qui se restructure, mais aussi un État-nation qui se mue en État-réseau au sein d'un hyper-capitalisme du sommet ${ }^{9}$. La direction du MEDEF est le représentant du niveau $1^{10}$ et ne représente pratiquement plus les intérêts du capital industriel en tant que tel sans pour cela représenter les intérêts d'un capital financier «déconnecté » de "l'économie réelle ». II ne défend les intérêts particuliers d'une fraction du patronat que dans la mesure où ils s'inscrivent dans les objectifs de la gouvernance stratégique fixée au niveau 1. Ces objectifs tendent vers l'unification globale du capital au sein d'une stratégie mondiale qui n'empêche pas la concurrence tout en faisant la part belle aux oligopoles. Tout doit être fluide, de la monnaie jusqu'à la force de travail puisque la production par le travail vivant n'est plus considérée que comme une prémisse du procès d'ensemble. De la même façon qu'on parlait auparavant des «faux frais » de la production on ne parle déjà plus de la production par le travail vivant que comme d'une activité comme une autre au sein de « la chaîne de création de valeur ».

\section{L'État et la gestion de la reproduction des rapports sociaux}

Les objectifs de l'État sont très globaux : reproduction des rapports sociaux et cohésion sociale d'un côté, assurer les meilleures conditions possibles au développement économique et à la compétitivité des entreprises de l'autre. C'est ce qui différencie le représentant $\mathrm{du}$ capital global de chaque capital particulier, fut-il une firme multinationale. En effet, l'État doit mener les deux opérations de front alors que pourtant chacune exerce une contrainte sur l'autre. Ce n'est pas le cas de chaque capital particulier qui essaie, depuis les restructurations de ces vingt dernières années, de s'émanciper de la première contrainte, en la renvoyant soit sur ses concurrents (la recherche de la compétitivité-prix) soit sur l'État (solidarité nationale et traitement social du chômage).

Dans cette lecture, la « prime pour l'emploi » n'est pas un cadeau fait aux patrons, comme on peut le lire un peu partout dans les journaux d'extrême gauche. Il s'agit d'une stratégie politique qui prend en compte la caducité de la loi de la valeur et les simplifications abusives qui se cachent derrière les théories de l'exploitation. Elle cherche donc à diversifier ses réponses en fonction de l'éclatement des références et des statuts produit par la révolution du capital : 
44 - d'un côté, en s'adressant majoritairement à ceux qui sont dans le circuit du travail, à un degré ou un autre, cette stratégie s'inscrit dans le refus de l'État de cautionner un système d'assistance aux pauvres supposés oisifs. Il faut récompenser le salarié-pauvre bien méritant en lui montrant que d'abord il a tout intérêt à continuer à travailler et qu'ensuite, de toute façon, il n'a pas le choix.

45 - d'un autre côté, pourtant, cette restructuration n'unifie pas davantage une classe de salariés enfin devenue homogène comme dans l'ancienne perspective de la "moyennisation », mais elle redistribue les inégalités aussi bien au niveau vertical et hiérarchique de la propriété et du travail (ce qui prévaut n'est plus forcément l'opposition propriétaire des moyens de production/non-propriétaires mais plutôt l'opposition dirigeants/exécutants ${ }^{11}$ ) qu'au niveau horizontal (inclus/exclus du travail, du logement, des papiers, des échanges).

C'est aussi la manière qu'à la société capitalisée, d'exprimer la fin d'une société de classes dont le modèle d'origine fondé sur l'opposition entre deux blocs monolithiques finalement rassurant quand ces blocs-classes pratiquent la coexistence pacifique, laisse place à la peur d'une nouvelle "question sociale ». Il n'est donc pas étonnant que le Pouvoir y réponde par l'antienne de remettre le travail au centre de la société, mais d'un travail qui ne garderait que sa fonction de discipline ou de compétition et non plus sa fonction productive qui devient accessoire. Les dominants ont bien conscience que tout cela ne peut rentrer dans le cadre strict du salariat. La nouvelle idéologie du travail se détache donc chaque jour un peu plus de la réalité du travail salarié comme elle s'était déjà détachée de la figure du travailleur productif, pour recouvrir tout type de travail et laisser penser que le travail peut redevenir « libre » dans l'idéal de « monter sa boîte » et la figure du « gagneur ». Le « travailler plus pour gagner plus » de Sarkozy en est déjà une version diluée par les désillusions des vingt années précédentes (l'ère Tapie).

47 À l'époque où la valorisation s'effectuait essentiellement par le travail vivant, la survaleur produite à l'aide de ce dernier était fondamentale et la dimension « sociale » du système de salariat n'était qu'induite. Pour toute une frange de surnuméraires potentiels, aujourd'hui c'est la situation inverse qui prédomine: les «forteresses ouvrières » survivantes (par exemple dans l'automobile) finissent à peine de dégraisser que les nouveaux secteurs (banques, assurances, médias et informatique) licencient déjà à tout va. Pour reproduire les rapports sociaux il faut alors créer des emplois dont, il y a quelques temps encore, il serait venu à personne l'idée de les appeler du travail.

La discipline du travail doit s'imposer quand le travail vivant n'a plus de valeur mesurable en temps de travail (comment déterminer sa part dans la création de valeur?) ou en valeur intrinsèque (quel est le niveau de salaire équivalent à la reproduction de la force de travail aujourd'hui pour rester dans la problématique marxiste ?), quand les identités de classe et les identités professionnelles disparaissent et qu'il ne reste plus que la course aux revenus et la résistance à l'atomisation sociale.

49 Cette discipline passe par de nouvelles règles, dont le pacte pour un retour à l'emploi (PARE) nous fournit un exemple. Mais ces règles doivent rester politiquement compatibles avec l'idée de bien commun, de cohésion sociale et autres balivernes consensuelles. Il en va du développement de cette nouvelle forme de capitalisme qui donne l'impression de vouloir s'émanciper de toute contrainte, mais qui en même temps ne le peut pas car elle doit continuer à se présenter sous l'apparence d'une légitimité 
supérieure requérant l'adhésion active ou passive du plus grand nombre à l'idéologie de l'entreprise-société protégée par l'existence, en dernier recours, de l'État.

Il y a bien une tentative de "refondation sociale », mais à l'état de projet, sans rien de définitif. C'est la différence avec l'époque des classes-sujets. Il n'y a plus de projet au sens fort, c'est-à-dire s'accompagnant d'une certaine Weltanschauung (pour la bourgeoisie) ou d'un sens millénariste (pour le prolétariat). Il ne faut donc pas élaborer des fictions sur un grand ordonnateur qui tirerait les ficelles à partir d'un « plan du capital », ce qui ne veut pas dire non plus qu'il faudrait alors se réfugier dans une vision structuraliste faisant la part belle au « capital-automate».

51 Tout cela doit nous amener à être très modeste quant à la vérité et à la portée heuristique de nos analyses ${ }^{12}$.

La question de la discipline du travail et par le travail n'est pas, comme je l'ai dit précédemment, une réponse directe aux pratiques anti-travail. Cette dimension répressive du travail n'était cependant pas absente pendant et immédiatement après la période des années 1960-1970. Mais la défaite du dernier assaut prolétarien ainsi que le processus d'inessentialisation de la force de travail ${ }^{13}$ qui s'est renforcé dans la période ultérieure des restructurations, donnent un autre sens à la crise actuelle du travail. Comme le disait Marx, «le mort saisit le vif », avec une tendance à la domination du travail mort sur le travail vivant et un processus de substitution capital/travail qui n'a pris toute son ampleur que depuis la fin du $\mathrm{XX}^{\mathrm{e}}$ siècle. Mais que le travail vivant ne soit plus vraiment au cœur de la production, cela ne signifie pas que le travail en tant que valeur soit mort. En tout cas, dans le doute beaucoup veulent le ranimer. C'est là qu'on retrouve la dimension disciplinaire dans la variété des positions travaillistes, que ce soit celles de Blair et Brown, du PS ou de la CFDT. Aujourd'hui, tout le discours qui prétend remettre le travail au centre de la société vise à discipliner la force de travail potentiellement sans emploi et celle dont le travail n'est justement qu'un emploi, un job. Il vise aussi à une remoralisation des salariés alors que tous les signaux envoyés par la société capitalisée mettent en lumière non pas une société du travail, mais généralisation des pratiques de prédation, de captage et de rente.

\section{Pour conclure}

On est passé d'un mouvement de critique du travail a des comportements individuels de refus qui peuvent s'expliquer par le peu d'intérêt (dans tous les sens du terme) que représente le fait de travailler; la difficulté qu'il y a à trouver un travail quand on ne correspond pas au modèle de l'employabilité. Malgré des positions plus radicales et plus théorisées telles celles du groupe CARGO en France ou celles des Chômeurs heureux en Allemagne, ce qui prédomine, c'est une critique passive ou des actions ponctuelles comme celles des comités de chômeurs en France en 1998, comme le montre encore la tendance dominante à désamorcer des conflits possibles par plans sociaux successifs (aujourd'hui encore en Italie par exemple avec des plans de pré-retraites négociés avant l'arrivée au pouvoir du gouvernement d'experts de M. Monti).

La limite des pratiques anti-travail des années 1960-1970 et les erreurs d'interprétation qui s'en sont suivies, c'est de n'avoir appréhendé le travail que comme aliénation (la "critique artiste» dont parlent Boltanski et Chiapello) et exploitation (la critique ouvrière marxiste) et non pas comme une contradiction de l'activité humaine (le travail 
en général comme contradiction de l'activité générique $\left.{ }^{14}\right)$. Nous avons donc perçu le déclin de ces pratiques seulement comme le retournement contre-révolutionnaire d'un cycle révolutionnaire qui s'achevait, sans tenir compte du fait que la situation antérieure ne se reproduirait plus. En effet, les cycles analysés par Marx où révolution et contrerévolution se succédaient sont aujourd'hui inopérants parce que le rapport social capitaliste a réussi à englober ses deux pôles, le capital et le travail, en détruisant les sources originelles des antagonismes de classes et particulièrement la dépendance réciproque entre les deux pôles. La société capitalisée s'autonomise de son rapport social dans la mesure où son pôle capital a tendance à s'auto-présupposer sans son pôle travail et cela aussi bien à travers une anticipation ex ante du profit ${ }^{15}$ en fonction de toute la chaîne de valeur et non plus simplement de la valeur-travail; qu'à travers sa financiarisation et l'évanescence de la valeur ${ }^{16}$.

Il n'y a donc pas de contre-révolution du capital en opposition à ce qui serait la révolution d'un prolétariat, mais une révolution du capital ou ce que certains appellent une révolution anthropologique (P. P. Pasolini).

La critique du travail est maintenant l'œuvre du capital lui-même. Certes le travail subsiste encore, comme l'entrepreneur capitaliste d'ailleurs, mais à l'état de résidu dont on ne sait quoi faire. Il n'est plus moteur dans la création de la valeur. Il est travailfonction dans un système d'attribution des revenus. Le stade actuel atteint par la contradiction, c'est que le revenu est de plus en plus socialisé, de moins en moins lié à un travail concret exercé, mais qu'il doit encore s'inscrire dans le salariat sous peine de blocage de sa logique.

Juin 2012

\section{NOTES}

1. Sur cette distinction, cf. K. Marx : Le sixième chapitre inédit du capital, Bourgois, coll 10/18. Voir aussi nos articles «Quelques réflexions sur domination formelle et domination réelle » dans le $\mathrm{n}^{\circ}$ 14 de Temps critiques disponibles ici http://tempscritiques.free.fr/spip.php? page=numero\&id_numero $=14$

2. C'est toute l'ambiguïté de Marx qui, dans les Manuscrits de 1844, énonce à la fois une critique de l'aliénation dans le travail et une définition du travail comme essence de l'homme en tant qu'activité générique, au-delà donc de ses formes particulières.

3. Pour une critique de cette position on peut se référer à J.P. Mandosio: Dans le chaudron du négatif, L'Encyclopédie des Nuisances, 2003 et D'or et de sable, même éditeur, 2008.

4. Cf. le titre du $n^{\circ} 13$ de la revue Temps critiques, 2003.

5. Le texte de Zerzan: «Un conflit décisif, les organisations syndicales combattent la révolte contre le travail » est traduit et reproduit dans Échanges de décembre 1975 avec la réponse de Reeve.

6. Cf. le $\mathrm{n}^{\circ} 12$ de Temps critiques (hiver 2001) et plus particulièrement mon article : « Neo-luddisme et résistances ouvrières ", p. 16-26.

7. Cf. le $\mathrm{n}^{\circ} 15$ de Temps critiques et Après la révolution du capital, éd. L'Harmattan, 2007. 
8. Ce point a été particulièrement développé par le courant néo-opéraïste autour de Lazzarrato et Negri, dans la revue Futur antérieur. Nous en avons fait une critique dans «Sur deux méprises à propos des intermittents du spectacle ». Temps critiques, $\mathrm{n}^{\circ} 14$, p. 70-71.

9. A ce sujet on peut se reporter aux numéros 15 et 16 de Temps critiques.

10. Cette caractérisation en trois niveaux est développée dans l'article «Quelques précisions sur capitalisme, capital, société capitalisée ", Temps critiques $n^{\circ} 15$. Disponible en ligne http:// tempscritiques.free.fr/spip.php?article206

11. Cf. le rôle joué par le développement des stock-options et des bonus dans ce schéma d'une "désalarisation » partielle par le haut.

12. Si on prend par exemple l'analyse de la loi sur les 35 heures, bien malin aujourd'hui qui pourrait en lire les effets à moyen terme. Si on regarde ce qui se passe à La Poste, cela a entraîné la création d'un nombre très important de CDI, mais avec une aggravation des conditions de travail pour certaines catégories (les facteurs et le problème des samedis, flexibilité accrue avec semaine de quatre jours ou quatre jours et demi dans les bureaux... mais avec rigidité accrue dans la prise et la fin de poste !) Où est la lisibilité de tout cela?

13. II ne s'agit pas de savoir combien il y a de travailleurs productifs, si le nombre baisse ou augmente, en France ou dans le monde, absolument ou relativement, comme il est envisagé dans les discussions entre marxistes, mais de voir que cette force de travail devient périphérique dans la production de valeur, inessentielle donc, seule position qui peut permettre de faire immédiatement le lien, au niveau théorique, entre lutte des salariés et luttes des chômeurs.

14. C'était pourtant la voie tracée par Marx dans ses Manuscrits de 1844.

15. L'exemple le plus souvent cité est celui du taux de retour sur investissement qui est attendu (autour de 15\%) qui contraint les entreprises dont le taux de profit tourne souvent autour de 5\%, à faire les efforts nécessaires pour réduire le différentiel, y compris en licenciant du personnel.

16. Cf. J. Guigou et J. Wajnsztejn : L'évanescence de la valeur, L'Harmattan, 2004.

\section{INDEX}

Mots-clés : critique du travail, correspondance, artistes, socialisme vert, travailleurs culturels, Wertkritik, Gorz André, dialogue, Vincent Jean-Marie, écologie, modèles alternatifs, Marx Karl, penser le Capital, ethos du travail, Eglise Orthodoxe, salariat, domination

\section{AUTEUR}

\section{JACQUES WAJNSZTEJN}

Ecrivain, co-directeur de la revue Temps critiques (www.tempscritiques.net). 